\title{
Identification of Filamin-A and -B as potential biomarkers for prostate cancer
}

Aim: A novel strategy for prostate cancer ( $\mathrm{PrCa}$ ) biomarker discovery is described. Materials \& methods: In vitro perturbation biology, proteomics and Bayesian causal analysis identified biomarkers that were validated in in vitro models and clinical specimens. Results: Filamin-B (FLNB) and Keratin-19 were identified as biomarkers. Filamin-A (FLNA) was found to be causally linked to FLNB. Characterization of the biomarkers in a panel of cells revealed differential mRNA expression and regulation. Moreover, FLNA and FLNB were detected in the conditioned media of cells. Last, in patients without PrCa, FLNA and FLNB blood levels were positively correlated, while in patients with adenocarcinoma the relationship is dysregulated. Conclusion: These data support the strategy and the potential use of the biomarkers for PrCa.

Lay abstract: The goal of this study was to use a novel strategy that combines biological outputs with Bayesian network learning to identify potential biomarkers for prostate cancer (PrCa). This methodology identified two proteins, filamin B and keratin-19, as potential biomarkers for PrCa. The network map also identified a direct linkage between filamin B and filamin A, which is a protein that has previously been identified as playing a role in PrCa etiology. The identified proteins were then validated by examining their levels in a panel of PrCa cell lines and in human plasma samples.

First draft submitted: 25 August 2016; Accepted for publication: 31 October 2016; Published online: 22 December 2016

Keywords: Bayesian network learning • biomarkers $\bullet$ FLNA $\bullet$ FLNB $\bullet$ KRT19 • prostate cancer

The prostate-specific antigen (PSA)-based blood test is used as a first-line assay for detecting prostate cancer (PrCa) [1,2]. However, due to its lack of accuracy it has led to over diagnosis and unnecessary aggressive interventions in patients with indolent disease. Thus, the US Prevention Services Task Force issued a recommendation against the use of PSA-based screening in 2012 [3]. Thus, there remains a critical unmet clinical need for identification of novel biomarkers to improve detection of PrCa.

Novel biomarkers that are currently in development for PrCa include the TMPRSS2$E R G$ fusion, $P C A 3$ and $A M A C R$, each of which have been described for use alone or use in combination to improve the diagnosis of PrCa [4]. However, a major disadvantage of these biomarkers is the need for core biopsy specimens for assessment [4]. Thus, while these tests alone or in combination with PSA are clinically useful [4], discovery and development of biomarkers that can be detected in biofluids or any other easily available matrix would provide an advantage over existing markers in development.

Proteomic profiling of the secretome of cancer cells is a promising strategy that is employed to identify novel biomarkers for various cancers including PrCa.
Niven R Narain'1, Anne R Diers' ${ }^{1}$, Arleide Lee ${ }^{1}$, Socheata Lao', Joyce Y Chan', Sally Schofield', Joe Andreazi', Rakibou Ouro-Djobo', Joaquin J Jimenez ${ }^{4}$, Tracey Friss', Nikunj Tanna', Aditee Dalvi', Sihe Wang ${ }^{2,3}$, Dustin Bunch $^{2,3}$, Yezhou Sun', Wenfang Wu', Khampaseuth Thapa', Stephane Gesta', Leonardo O Rodrigues ${ }^{1}$, Viatcheslav R Akmaev', Vivek K Vishnudas ${ }^{1}$ \& Rangaprasad Sarangarajan*,1

'Berg, LLC, Framingham, MA, USA 2Department of Laboratory Medicine, Cleveland Clinic, Cleveland, OH, USA 44195

${ }^{3}$ Department of Chemistry, Cleveland State University, Cleveland, OH, USA 44115

${ }^{4}$ Department of Dermatology, University of Miami Miller School of Medicine, Miami, FL, USA 33136

*Author for correspondence: rangaprasad.sarangarajan@berghealth. com

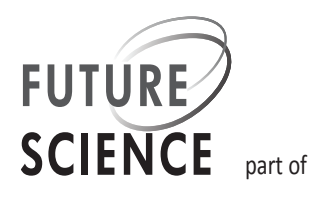


The 'secretome' is a term that was first presented by Tjalsma et al. based upon their findings from genome-wide screening of putative proteins that are secreted by Bacillus subtilis [5]. As secreted proteins may theoretically enter bodily fluids, their potential ability to be detected using noninvasive methods makes them a highly attractive target for biomarker discovery. Therefore, in the present report a novel data-driven systems biology-based approach was used to identify potential biomarkers for $\mathrm{PrCa}$ from interrogation of the secretome of PrCa cells (see Supplementary Figure 1 for schematic).

\section{Materials \& methods}

Materials

All chemicals were obtained from Sigma-Aldrich and of analytical grade unless otherwise noted (MO, USA). Methyltrienolone (R1881) was obtained from PerkinElmer (MA, USA).

\section{Cell culture}

Normal, human, primary prostate epithelial cells (HPrEC) (Lifeline Cell Technology, MD, USA) were maintained in ProstaLife media containing $6 \mathrm{mM}$ L-glutamine, $0.4 \%$ bovine pituitary extract, $1 \mu \mathrm{M}$ epinephrine, $0.5 \mathrm{ng} / \mathrm{ml} \mathrm{TGF} \alpha, 100 \mathrm{ng} / \mathrm{ml}$ hydrocortisone hemisuccinate, $5 \mu \mathrm{g} / \mathrm{ml}$ insulin and $5 \mu \mathrm{g} /$ $\mathrm{ml}$ apo-transferrin. LNCaP and PC-3 PrCa cell lines (Sigma-Aldrich) were maintained in RPMI 1640 and high glucose DMEM, respectively. DU145 PrCa cells were maintained in Eagle's Minimum Essential Medium (EMEM). Base media were supplemented with $10 \%$ fetal bovine serum and $1 \times$ Pen/Strep/ AmphoB. All cell lines were maintained in a humidified environment at $37^{\circ} \mathrm{C}$ under $5 \% \mathrm{CO}_{2}$. All cells lines were authenticated using short tandem repeat DNA profiling (Genetica DNA Laboratories, Inc., NC, USA). For treatment with R1881, cells were incubated in charcoal-stripped fetal bovine serumcontaining media for $24 \mathrm{~h}$ prior to exposure to R1881.

\section{Human plasma samples}

Residual lithium-heparin plasma was collected from patients with suspected $\mathrm{PrCa}$ (see Table 1 for patient demographics). The criteria for inclusion were: elevated PSA results $(\geq 2.6 \mathrm{ng} / \mathrm{ml})$, age $45-70$ years and $\geq 700 \mu \mathrm{l}$ of sample. Samples were excluded if patients had any cancer diagnosis prior to collection. Biopsy information was accessed 6-8 weeks after sample collection. The diagnosis of benign, prostatic intraepithelial neoplasia or adenocarcinoma was noted. A Gleason score was recorded for patients with prostate adenocarcinoma. All procedures were performed in accordance with the ethical standards of the responsible committee on human experimentation (institutional and national) and with the Helsinki Declaration of 1975, as revised in 2000 and 2008.

\section{Identification of potential biomarkers using a data-driven approach}

Cells were cultured in low $(5 \mathrm{mM})$ or high $(22 \mathrm{mM})$ glucose, with and without lactic acid $(12.5 \mathrm{mM})$, and at normoxia ( $21 \%$ oxygen) or hypoxia ( $2 \%$ oxygen) (see Supplementary Figure 1 for schematic). Conditioned media were harvested at 24 and $48 \mathrm{~h}$ for proteomics analysis [6], and used as inputs for the Bayesian network inference model.

\section{Proteomic analysis of conditioned media}

After cells were treated with various stimuli, $15 \mathrm{ml}$ of conditioned media were collected and frozen at $-20^{\circ} \mathrm{C}$ until use. Samples were thawed, then concentrated using a $30 \mathrm{kDa} \mathrm{MW}$ cutoff filter (Amicon UFC903008, EMD Millipore, MA, USA) prior to centrifugation for $18 \mathrm{~min}$ at $4000 \times g$ at $4^{\circ} \mathrm{C}$. Protein concentration was determined using the Bradford assay. Up to $50 \mu \mathrm{g}$ of protein was prepped using the filter-aided sample preparation method (Expedeon, CA, USA) and processed for proteomic analysis as previously described [6]. Briefly, samples were reduced by addition of $200 \mu \mathrm{l}$ of $8 \mathrm{M}$ urea and $10 \mathrm{mM}$ DTT (Sigma-Aldrich), vortexed for $30 \mathrm{~min}$ at room tempreture (RT), transferred to filter-aided sample preparation spin filters and spun down for $10 \mathrm{~min}$ at $14,000 \times g$. This process was repeated for an additional time. Sample alkylation was performed by adding $10 \mu \mathrm{l}$ of resuspended iodoacetamide and incubation at RT for $20 \mathrm{~min}$. Samples were centrifuged and washed twice with $100 \mu \mathrm{l}$ of $8 \mathrm{M}$ urea and once with $100 \mu \mathrm{l}$ of $50 \mathrm{mM}$ ammonium bicarbonate. For digestion, $2 \mu \mathrm{g}$ of trypsin (Sigma-Aldrich) was added to each sample. Samples were incubated at $37^{\circ} \mathrm{C}$ overnight with gentle linear shaking then eluted the next day with $40 \mu \mathrm{l}$ of ammonium bicarbonate prior to centrifugation followed by elution with $110 \mu$ l of optima water. Samples were then dried down in a speed vacuum for $1.5 \mathrm{~h}$ and desalted using Pierce C18 desalting spin columns (Thermo Fisher Scientific, MA, USA). The desalted samples were dried down and resuspended in $20 \mathrm{mM}$ ammonium formate.

Approximately $5 \mu \mathrm{g}$ of sample was injected and run on a Waters 2D NanoAquity HPLC system coupled with a Thermo Q Exactive Plus Mass spectrometer (Thermo Fisher Scientific). The LC ran five fractions with 90 min gradients while the mass spectrometer 
Table 1. Patient demographics.

\begin{tabular}{|lclll|}
\hline Condition & N & $\begin{array}{l}\text { Mean age } \\
(\text { range })\end{array}$ & $\begin{array}{l}\text { Mean PSA }( \pm \\
\text { SEM) }\end{array}$ & Mean number of biopsies \\
\hline Benign & 14 & $60.86(50-69)$ & $6.3( \pm 1.3)$ & $9( \pm 1.0)$ \\
\hline Benign, intraepithelial neoplasia & 4 & $62.3(57-69)$ & $6.2( \pm 0.9)$ & $12.25( \pm 3.8)$ \\
\hline Benign total & 18 & & & \\
\hline Adenocarcinoma total & 28 & $61.2(50-69)$ & $15.7( \pm 5.0)$ & $10.2( \pm 1.1)$ \\
By Gleason Score: & 7 & & & \\
6 & 9 & & \\
7 & 1 & & \\
8 & 2 & & \\
9 & 46 & & \\
Total & 9 & & \\
\hline PSA: Prostate-specific antigen; SEM: Standard error of the mean. &
\end{tabular}

(MS) collected full MS and MS/MS (Top 20) data over $500-1800 \mathrm{~m} / \mathrm{z}$. The acquired MS/MS spectra were analyzed with Proteome Discover 1.4 (Thermo Fisher Scientific) using a SequestHT search engine and a Human fasta database. Search parameters included $20 \mathrm{ppm}$ for MS tolerance, $0.02 \mathrm{Da}$ for $\mathrm{MS}^{2}$ tolerance and full trypsin digestion, which allows for up to four missed cleavages. Carbamidomethylation (C) was set as the fixed modification. Oxidation (M) and deamidation (NQ) were set as dynamic modifications. Peptides and protein identifications were filtered to allow a $99 \%$ confidence level of protein identification ( $1 \%$ false discovery rate).

\section{Bayesian network inference}

Bayesian networking is a mathematical methodology developed for characterization of a multivariate system of random variables. The Bayesian networks approach is a framework where each particular factorization and the choice of parameters are a distinct probabilistic model of the process that created the observed experimental data [7]. A model ensemble of networks was built by using a Bayesian network learning and simulation software that combined the shotgun isobaric tag for relative and absolute quantitation (iTRAQ) proteomics data and the functional outputs [8]. The functional outputs include results of low and high glucose conditions, presence of lactic acid and oxygenation status [8]. Individual node in silico knockdown simulations were analyzed to refine the graph model. The expression level of each protein was reduced one at a time and the posterior distributions of all downstream nodes were statistically compared with their baseline distributions by calculating the c-statistic and the fold difference between the posterior and baseline distribution means.
The sub-networks linked to the functional variables were analyzed by determining their Burt's constraint metric [9]. This measure calculates the extent to which nodes are connecting to unconnected modules and the relationship redundancy within each of these modules. Nodes with lower Burt's constraint score are more likely to have a stronger effect on the network structure once it is bridged between nonredundant modules. The inferred molecular interaction networks represent localized casual pathways that may drive cytotoxicity so that the nodes with the lowest Burt's constraint metric are potential biomarkers.

\section{Quantitative real-time PCR}

Total RNA was extracted using a Qiagen RNAeasy kit per manufacturer's protocol (Qiagen, MD, USA). First-strand cDNA synthesis was performed using 1 $\mu \mathrm{g}$ of RNA using the Invitrogen high-capacity cDNA reverse transcription kit (Thermo Fisher Scientific). Quantitative real-time PCR was subsequently carried out using the Bio-Rad CFX384 Real Time System (Bio-Rad, CA, USA). PCR primers for specified genes were used with iQ SYBR Green Supermix (Bio-Rad). TATA-binding protein primers were used with TaqMan Universal PCR Master Mix (Invitrogen) and served as a house-keeping control gene. All results were normalized to TATA-binding protein using the $\Delta \mathrm{Ct}$ method.

\section{Detection of PSA in human serum samples by} electrochemiluminescence immunoassay

Total PSA testing was performed using the Elecsys total PSA immunoassay reagent (Roche Diagnostics, IN, USA), which measures total (free + complexed) PSA, on a Roche Cobas E602 instrument (Roche Diagnostics). The assay was performed per manu- 
facturer's recommendations. Briefly, $20 \mu \mathrm{l}$ of sample was incubated with biotinylated monoclonal PSAspecific antibody and a monoclonal PSA-specific antibody labeled with ruthenium complex Tris $\left(2,2^{\prime}\right.$ bipyridyl)ruthenium(II)-complex (Ru(bpy)), which react to form a sandwich complex. Streptavidincoated microparticles were then added to form a complex with biotinylated-labeled antibody. The reaction mixture was then aspirated into the measuring cell where the microparticles were then magnetically captured onto the surface of the electrode. Any unbound substance was subsequently removed with ProCell/ProCell M. A voltage was then applied to the electrode, which induced chemiluminescent emission that was measured by a photomultiplier. Results were then determined via a calibration curve generated by the instrument using a two-point calibration and a master curve provided by the reagent barcode.

\section{Detection of filamin-B in human serum samples by ELISA}

Microtiter polystyrene 96-well plates (R\&D Systems, MN, USA) were coated with $100 \mu$ l per well of $14 \mu \mathrm{g} / \mathrm{ml}$ coating antibody solution made with Antibody 5 (3F10 Antibody Group) diluted in phosphate buffered saline (PBS) (R\&D Systems). Specifically, the plates were incubated overnight, washed with wash buffer $(0.05 \%$ Tween-20 in PBS, pH 7.2-7.4) and blocked with $1 \%$ bovine serum albumin (BSA) in PBS, $\mathrm{pH}$ 7.2-7.4. During the assay, $50 \mu \mathrm{l}$ of assay diluent was added to all wells followed by $100 \mu \mathrm{l}$ of standard, control and sample per well. The standard was full-length recombinant human protein (Genscript, NJ, USA) in the range of $0.156-10 \mathrm{pM}$. The plate was securely covered with a plate sealer and incubated for $2 \mathrm{~h}$ at RT on an orbital shaker set at $180 \pm 20 \mathrm{rpm}$. The plates were washed with $400 \mu \mathrm{l}$ of wash buffer for a total of four times. Plates were then incubated with $200 \mu$ l of detection antibody (biotinylated filamin-B [FLNB]-specific monoclonal antibody, $0.1 \mu \mathrm{g} / \mathrm{ml}$; R\&D Systems) and incubated for $2 \mathrm{~h}$ at RT on an orbital shaker set as specified above and followed by washes. Samples were then incubated in $200 \mu \mathrm{l}$ of Streptavidin-horse radish peroxidase (HRP) for $30 \mathrm{~min}$, then washed prior to addition of $200 \mu \mathrm{l}$ of substrate solution (a 1:1 mixture of $\mathrm{H}_{2} \mathrm{O}_{2}$ and tetramethylbenzidine) followed by incubation for $30 \mathrm{~min}$ at RT in the dark. The reaction was terminated with $50 \mu \mathrm{l}$ of $2 \mathrm{~N}$ sulfuric acid (R\&D systems) and the plate was read at 450-540 nM using a BioTek plate reader (BioTek, VT, USA) within $30 \mathrm{~min}$. A $\log -\log$ curve fit was used to calculate sample concentrations.

\section{Detection of filamin-A in human serum samples by ELISA}

Microtiter polystyrene 96-well plates (R\&D Systems) were coated with $250 \mu \mathrm{l}$ per well of $6 \mu \mathrm{g} / \mathrm{ml}$ coating antibody solution made with Antibody 4 (Berg ID 112597, Antibody Group) diluted in PBS. For the coating process, the plates were incubated overnight, washed with wash buffer $(0.05 \%$ Tween 20 in PBS, $\mathrm{pH}$ 7.2-7.4) and blocked with $1 \%$ BSA in PBS, $\mathrm{pH}$ 7.2-7.4. During the assay, $100 \mu \mathrm{l}$ of assay diluent was added to all wells followed by $50 \mu$ of standard, control and sample per well. The standard was fulllength recombinant human protein (Genscript, Berg ID 112054) in the range of $3.125-200 \mathrm{ng} / \mathrm{ml}$. The plate was sealed and incubated for $2 \mathrm{~h}$ at $\mathrm{RT}$ on an orbital shaker set at $150 \mathrm{rpm}$. The plates were washed with $400 \mu \mathrm{l}$ of wash buffer for a total of four times using a plate washer. Plates were then incubated with $200 \mu \mathrm{l}$ of detection antibody (biotinylated filamin-A [FLNA]-specific monoclonal antibody, $15 \mathrm{ng} / \mathrm{ml}$ ) for $2 \mathrm{~h}$ at RT on an orbital shaker set as specified above followed by washing the plate. Plates were then incubated with $200 \mu \mathrm{l}$ of Streptavidin--HRP for $30 \mathrm{~min}$, washed and then incubated in $200 \mu \mathrm{l}$ of substrate solution (1:1 mixture $\mathrm{H}_{2} \mathrm{O}_{2}$ and tetramethylbenzidine) for $30 \mathrm{~min}$ at RT, protected from light. The reaction was terminated with $50 \mu \mathrm{l}$ of $2 \mathrm{~N} \mathrm{H}_{2} \mathrm{SO}_{4}$ and the plate was read within 30 min using a microplate reader at 450 $\mathrm{nm}$ with a $540 \mathrm{~nm}$ correction. A $\log -\log$ curve fit was used to calculate sample concentrations.

\section{Statistical analysis}

Proteomics data were processed by parsing the number of peptide spectral matches (PSMs) of each peptide from a spreadsheet generated by the instrument. Only PSM numbers whose q-values were $<0.01$ were used. Peptides from nonhuman proteins were removed. PSM numbers for same peptide sequences with different modifications were combined. Total PSM number of each sample was scaled to the median of total PSM number of all samples. $\chi^{2}$ test was used for differential analysis on each peptide across conditions. Since each condition has the same number of replicates, sum of scaled PSM numbers for each group was used in a $\chi^{2}$ test. p-value was corrected to false discovery rate for multiple tests.

All other data were analyzed using GraphPad Prism (CA, USA). For comparison between two groups Student's t-test were performed. For comparisons with three or more groups one-way analysis of variance (ANOVA) followed by Dunnet's posthoc analysis for multiple comparisons was performed. A p-value of 0.05 was deemed significant. Correlations were calculated using Pearson's r. 


\section{Results}

Selection of candidate biomarkers

In multiple types of cancers, metabolic and redox imbalances play a critical role in regulating cell signaling events, which in turn activates survival pathways, disrupts cell-death signaling and increases cell proliferation, and thereby promote a malignant phenotype (reviewed in $[10,11])$. A combined approach encompassing in vitro perturbation, high-throughput proteomics of conditioned media and in silico sub-delineation of causal networks and ranking of molecules using Burt's constraint score was used to identify potential causal molecules of PrCa. FLNB and Keratin-19 (KRT19) were identified as very important nodes that were causally influenced by culture conditions of hypoxia and lactic acid in the metastatic prostate cancer cell line, PC-3 (Table 2). The Burt's constraint score metric of FLNB and KRT19 are ranked at the top of the list (Table 2 \& Supplementary Table 2). Of interest, FLNB showed a causal link to another filamin protein, FLNA (Supplementary Figure 2). Although FLNA was ranked at 52 (Table 2 \& Supplementary Table 2), given its causal link to FLNB and the literature supporting its role in $\mathrm{PrCa}$ [12-14], FLNA was further studied to biologically validate the in silico findings and to examine the relationship between FLNA and FLNB.

\section{Characterization of mRNA expression of biomarkers in vitro}

The identification of the molecules described above was derived from conditioned media of PC-3 cells (Supplementary Figure 1). Therefore, additional $\mathrm{PrCa}$ cell lines, LnCAP (androgen sensitive) and DU145 (androgen insensitive), were examined. Notably, the inclusion of an additional androgen-insensitive cell line that is less aggressive than PC-3 allowed us to compare across cell lines the basal mRNA expression and/ or secretion abilities of the potential biomarkers. The basal mRNA expression levels of FLNA, FLNB and KRT19 were characterized and compared with HPrEC (Figure 1). In LnCAP cells, FLNA and FLNB mRNA levels were significantly less than HPrEC. In DU145 cells FLNB mRNA levels were comparable to levels in HPrEC cell lines, while FLNA mRNA levels were significantly decreased compared with HPrEC cell lines. In PC-3 cells, mRNA levels of FLNB were lower than in $\mathrm{HPrEC}$, but there was no difference in FLNA mRNA levels between PC-3 and HPrEC. KRT19 mRNA levels were not significantly different across cell lines. These data demonstrate variation in mRNA expression of FLNA, FLNB and KRT19 across primary and PrCa cell lines. Moreover, these data suggest that differences in mRNA expression of the biomarkers across cell lines do not appear to be related to aggressiveness or androgen dependency.

\section{Transcriptional regulation of biomarkers by} prostate cancer-relevant stimuli in vitro

A number of microenvironmental cues are known to contribute toward $\mathrm{PrCa}$ etiology and progression including hypoxia, inflammatory stimuli and androgens $[15,16]$. In response to hypoxia a modest increase in FLNA mRNA expression (1.85-fold) was observed in LnCAP cells, but with no significant effect in DU145 and PC-3 cells (Figure 2A). FLNB expression was unaltered by hypoxia, while KRT19 mRNA was induced by hypoxia in LnCAP and PC-3 cells. Consistent with previous reports [17], PSA expression was markedly increased in response to hypoxia only in LnCAP cells. Expression of select hypoxia-responsive genes (GLUT-1, LDHA and Eno1) was used as a positive control to confirm increased mRNA expression of these genes across cell lines and thus activation of canonical hypoxic responses in all cell types (Supplementary Figure 3A).

$\mathrm{TNF} \alpha$ is an inflammatory stimulus known to drive $\operatorname{PrCa}$ development [18]. The expression levels of FLNA, FLNB, and KRT19 were not regulated by TNF $\alpha$ exposure in any cell type (Figure 2B). In contrast, PSA expression was significantly decreased by TNF $\alpha$ exposure in LnCAP cells, consistent with previous reports that androgen receptor (AR) signaling is suppressed by inflammation [19]. Similarly, when LnCAP, DU145 and PC-3 cells were treated with the R1881, FLNA, FLNB and KRT19 were not significantly affected, while $P S A$ expression was significantly altered in LnCAP cells (19-fold increase; Figure 2C), which is expected for an AR target gene [20]. Notably, the treatments of androgen-insensitive cells with androgen stimuli were included as negative controls to confirm the lack of ability to induce mRNA expression of PSA. In these cell lines, none of the potential biomarkers were induced by R1881, as expected for androgen-insensitive cells. The expressions of inflammatory/NFk $\beta(M C P-1, I L-6$ and COX-2) and androgen-(TMPRSS2) responsive genes were generally upregulated across cell lines and included to confirm activation of canonical inflammatory- and androgenresponses after TNF $\alpha$ and R1881 exposure, respectively (Supplementary Figure 3). Together, these data demonstrate that the regulation of FLNA, FLNB and KRT19 by PrCa-relevant stimuli is unlike PSA, suggesting their transcriptional regulation occurs through pathways independent of PSA.

\section{Secretion of biomarkers in vitro}

The initial identification of FLNA, FLNB and KRT19 as causal markers of PrCa was derived from the secretome of PC-3 cells. Therefore, whether the biomarkers could be detected in conditioned media 


\begin{tabular}{|c|c|c|c|c|c|c|}
\hline \multirow{2}{*}{$\begin{array}{l}\text { Burt's } \\
\text { measures }\end{array}$} & \multicolumn{3}{|c|}{ Score } & \multicolumn{3}{|c|}{ Rank } \\
\hline & FLNA & FLNB & KRT19 & FLNA & FLNB & KRT19 \\
\hline Closeness & $1.43 \mathrm{E}-05$ & $1.53 \mathrm{E}-05$ & $1.57 \mathrm{E}-05$ & 56 & 2 & 1 \\
\hline Constraint & 0.500 & 0.077 & 0.063 & 52 & 2 & 1 \\
\hline Degree & 2.000 & 13.000 & 16.000 & 52 & 2 & 1 \\
\hline
\end{tabular}

of LnCAP and DU145 cell lines was examined (Figure 3). FLNA and FLNB were detected in conditioned media from all cell types. KRT19 was detected in conditioned media from DU145 and PC-3 cells, but not in LnCAP cells. Together, these data indicate that KRT19 can only be detected in the secretome of DU145 and PC-3 PrCa cell lines, while FLNA and FLNB can be detected from the secretome of all PrCa cell lines examined.

\section{Assessment of plasma FLNA \& FLNB levels}

Given the ability to detect FLNA and FLNB in the secretome of all $\mathrm{PrCa}$ cell lines (Figure 3 ) and the in silico findings of a causal relationship between FLNA and FLNB (Supplementary Figure 2), FLNA and FLNB levels were assessed in human plasma samples from patients with suspected $\mathrm{PrCa}$ to determine proof-of-concept for detection in blood.

Patient demographics are shown in Table 1. There were no significant differences in age, PSA and mean number of biopsies between patients diagnosed with benign cases compared with those with adenocarcinoma. Notably, both FLNA and FLNB proteins were detected in plasma samples (Figure 4). However, there were no significant differences between groups in FLNA (mean \pm SEM $=182.5 \pm 15.22$ vs $168 \pm$ 13.39 for patients with benign vs adenocarcinoma, respectively) and FLNB $(0.444 \pm 0.06$ vs $0.663 \pm 0.15$ for patients with benign vs adenocarcinoma, respectively) (Figure 4A). As level of PSA is the current method utilized in the clinic for initial assessment of patients with suspected PrCa, PSA levels were analyzed to determine the ability for PSA to distinguish patients with benign vs adenocarcinoma (Figure 4A). The scatterplot depicts individual variation in PSA levels within each group. There were no significant differences in PSA levels between patients with benign vs adenocarcinoma in the group of patients analyzed in this study $(p>0.05)$. This is not surprising given that PSA-based assays are known to lack specificity.

As in silico findings demonstrated a causal relationship between FLNA and FLNB in PC-3 cells under conditions of hypoxia and lactic acid (Supplementary Figure 2), the relationship between FLNA and FLNB levels was examined in patients with benign cases and in patients with adenocarcinoma. Interestingly, in patients with benign cases, a strong positive correlation between FLNA and FLNB levels was observed (Pearson $r=0.765, p<0.001$; Figure 4B \& Supplementary Table 1). In contrast, in patients with adenocarcinoma, the relationship between FLNA and FLNB levels is diminished, as there is no significant correlation between them (Pearson $r=0.030805$; $p$

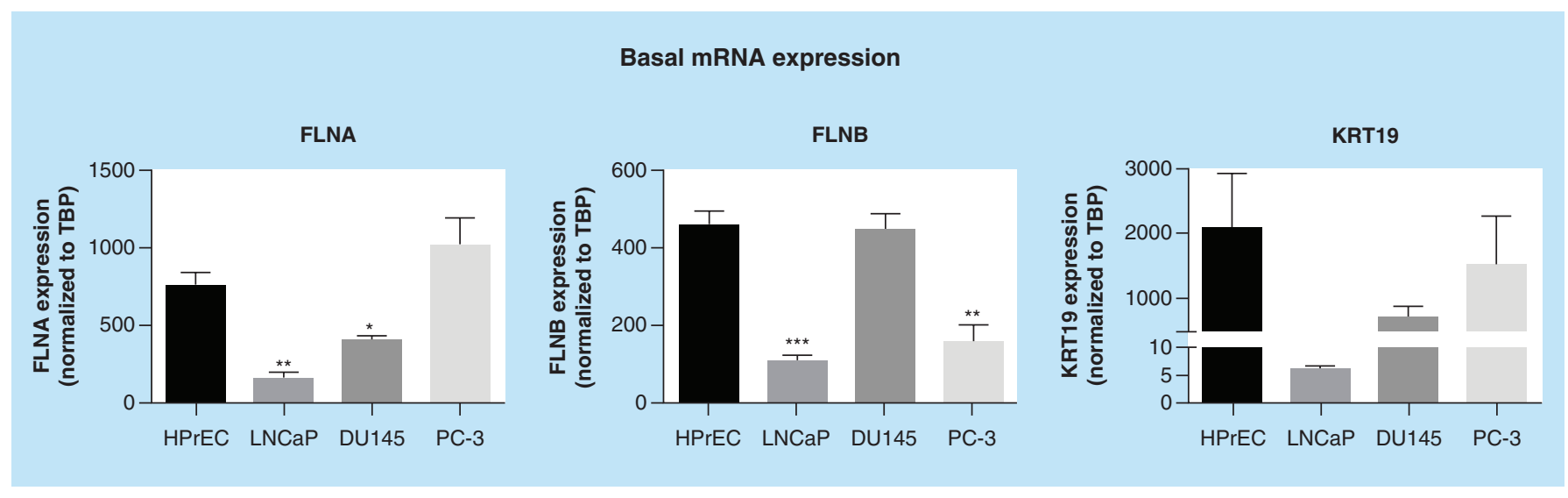

Figure 1. Basal mRNA expression of biomarkers in prostate cancer cells. Expression was assessed by quantitative RT-PCR and normalized to TBP. Data represent means + SEM, N = 3. ${ }^{*} p<0.05, * * p<0.01$ and $* * * p<0.001$ compared with HPrEC. HPrEC: Normal, human, primary prostate epithelial cells; FLNA: Filamin-A; FLNB: Filamin-B; KRT19: Keratin-19; RT-PCR: Real-time PCR; SEM: Standard error of the mean; TBP: TATA-binding protein. 

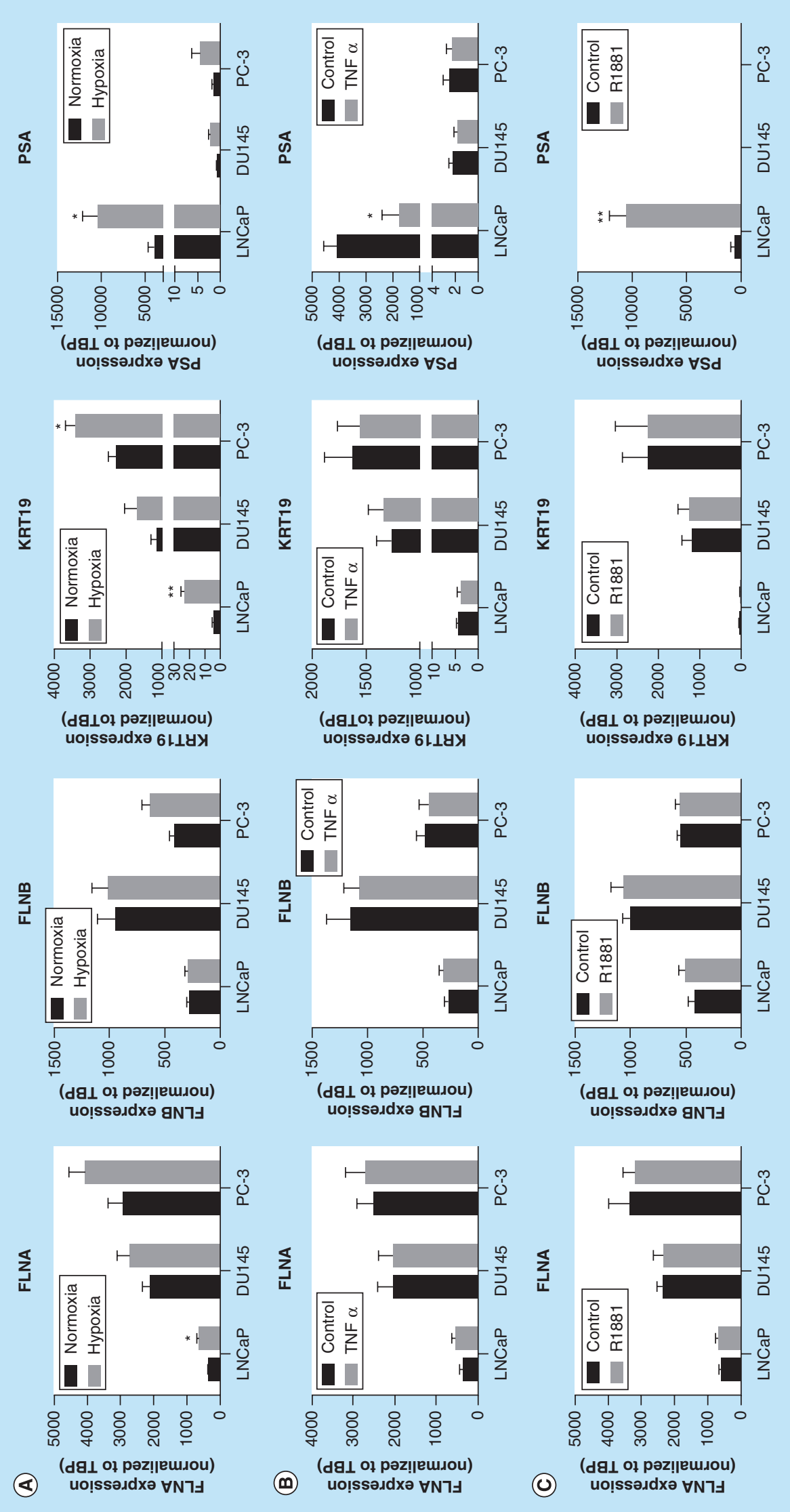

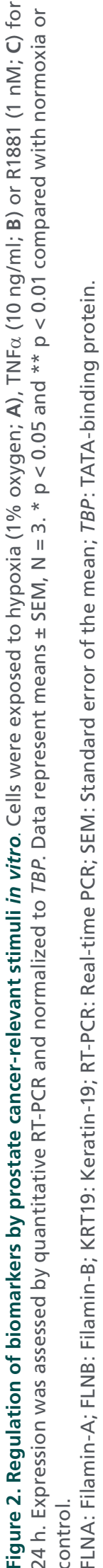




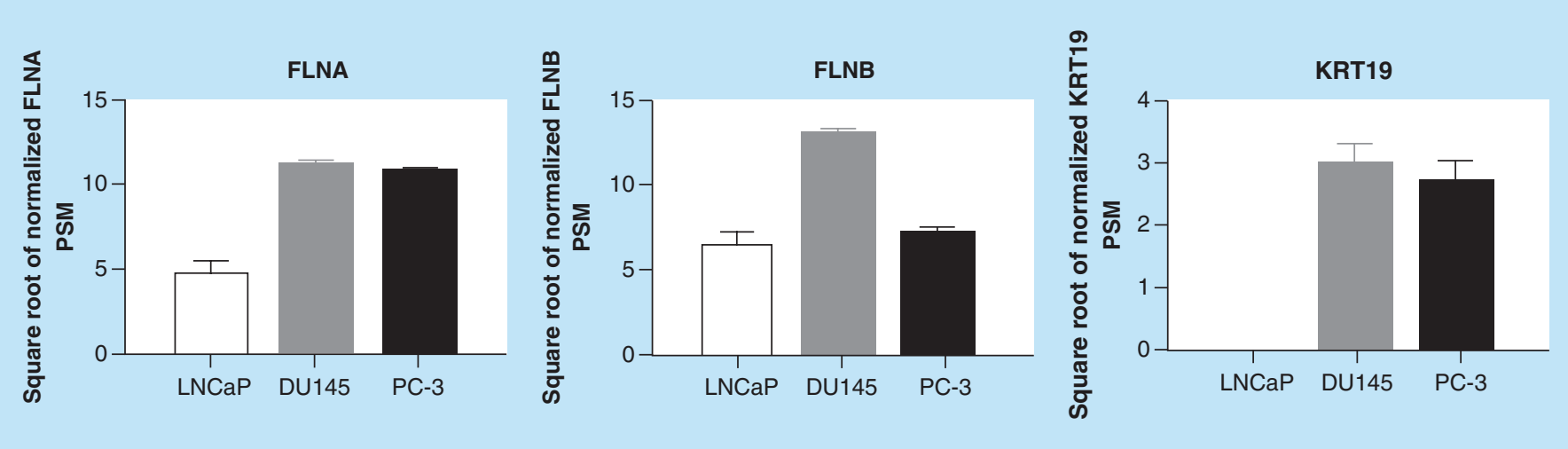

Figure 3. Secretion of biomarkers from prostate cancer cells. Conditioned media from cells were harvested and proteomic analysis was performed. Data represent means $\pm \mathrm{SEM}, \mathrm{N}=3$.

FLNA: Filamin-A; FLNB: Filamin-B; KRT19: Keratin-19; PSM: Peptide spectral match; SEM: Standard error of the mean.

$>0.05$; Figure 4B \& Supplementary Table 1). It must be noted that three samples were excluded from the analysis in patients with adenocarcinomas, as they were identified as outliers using the robust regression and outlier removal (ROUT) method $(\mathrm{Q}=$ $1 \%)$. Notably, inclusion of outliers did not affect the results (Pearson $\mathrm{r}=-0.052 ; \mathrm{p}>0.05$ ). Additional analysis in patients with adenocarcinoma revealed that PSA levels were positively correlated with Gleason score (Pearson $r=0.7865 ; \mathrm{p}<0.0001$; Supplementary Table 1), and FLNA levels were negatively correlated with Gleason score (Pearson $r=$ $-0.3921 ; \mathrm{p}<0.05$; Supplementary Table 1). However, age was not correlated with any variable in patients with benign cases or adenocarcinoma.

\section{Discussion}

This report describes the use of a systems biology approach to identify potential biomarkers for $\mathrm{PrCa}$ and provides preliminary validation of this approach. Importantly, a novel finding is that both FLNA and FLNB are secreted and the ability to detect both markers in human biofluids provides a noninvasive means to detect $\mathrm{PrCa}$ in patients. In addition, the dysregulation in blood levels of the two markers in patients with adenocarcinoma implicates an important biological link between the filamin proteins in PrCa.

Identification of disease-specific biomarkers requires determining differentials; a process which entails appropriate use of bioinformatics. Here, a Bayesian network structure learning methodology was used to identify novel causal biomarkers of PrCa. While the advantage and theoretical methods for use of Bayesian networks in genomic and proteomic cancer studies have been described [21], few studies have been published that have demonstrated application with biological output using the combined approach presented here.
Clinical utility of molecular markers for disease characterization is dictated by the presence or absence of the markers in easily available biofluids, for example, blood and urine. Proteomic profiling of the secretome is a useful tool for identifying novel markers for diseases. The secretome includes an array of proteins involved in processes such as immune responses and matrix remodeling, which are important mechanisms involved in invasion and metastasis of cancer cells $[22,23]$. It should be noted that it is possible that the detection of FLNA, FLNB and KRT19 in the secretome of in vitro cultures could be the result of release of intracellular proteins from dead cells (necrotic or apoptotic). However, it is unlikely that the levels detected in the present report reflect that released from dead cells. To ensure this, culture conditions were maintained at a confluency that was not overloaded, which allowed for growth and limited death in the brief time period of culture. Second, the lower LOD of MS would be unable to detect the levels of proteins that are released from the few cells that die. Moreover, there is literature that supports that FLNA and FLNB proteins are secreted. FLNA has been detected in mucus from the skin of fish [24] and interestingly, in isolated rat aorta ex vivo stimulation with N(omega)-nitro-Larginine methyl ester-induced secretion of a C-terminal fragment of FLNA [25]. Last, FLNB has been reported to be detected in human plasma [26].

KRT19 is the smallest and atypical in its class of keratins and is altered in cancers including pancreatic, hepatocellular and breast cancer. Altered expression of KRT19 has been demonstrated in bone marrow of metastatic PrCa patients [27]. The absence of secreted KRT19 protein in LNCaP cells compared with that in androgen refractory cell lines suggests utility of KRT19 as a biomarker for differentiating aggressive metastatic forms of $\operatorname{PrCa}$, a concept that is supported by other studies [12,27]. 
FLNA and FLNB belong to a family of large actinbinding filamins and play a major role in cell migration, vascular development, extracellular signaling and activity of integrins [12]. Reduced expression of FLNC has been observed in prostate tissue of patients [28], and is one of seventeen genes screened in prostate tissue samples in the commercially available Oncotype DX PrCa assay. Prior studies have demonstrated a role for FLNA in normal prostate physiology and in PrCa [13-14,29]. For example, Sun et al. demonstrated decreased FLNA intracellular protein expression in prostate tumors that correlated with $\mathrm{T}$ stage, lymph node metastasis, clinic stage and Gleason score, but not with age or PSA concentration [14]. Similarly, here FLNA blood levels were found to be negatively associated with Gleason score. However, this study is the first to identify and report FLNB as a potential biomarker for PrCa. Most notably, this report describes that the relationship between FLNA and FLNB is dysregulated in patients with PrCa. It has been proposed that FLNA and FLNB have overlapping roles in cell function [12]. However, the findings presented here suggest a poten- tial nonoverlapping functional link between the two proteins.

It must be noted that variations in intracellular mRNA expression and protein secretion were observed for all three molecules across the PrCa cell lines. Notably, the observation that there was no differential in FLNA and KRT19 mRNA expression after exogenous androgen treatment (R1881) between LnCAP and the androgen-insensitive, DU145 and PC-3 cell lines suggest that transcriptional regulation is independent of androgen sensitivity. Note, we confirmed androgen sensitivity of the cell lines by stimuli-dependent regulation of PSA expression in the cell lines and the regulation of canonical pathways. Moreover, the only stimulus that affected transcriptional regulation of the potential biomarkers was hypoxia in LnCAP and PC-3 cells, which suggests that the regulation of FLNA, FLNB and KRT19 by PrCa-relevant stimuli is unlike PSA.

In this report we focused on characterization of the potential biomarkers in PrCa cell lines, but it must be noted that FLNA has been described to be dysregu-
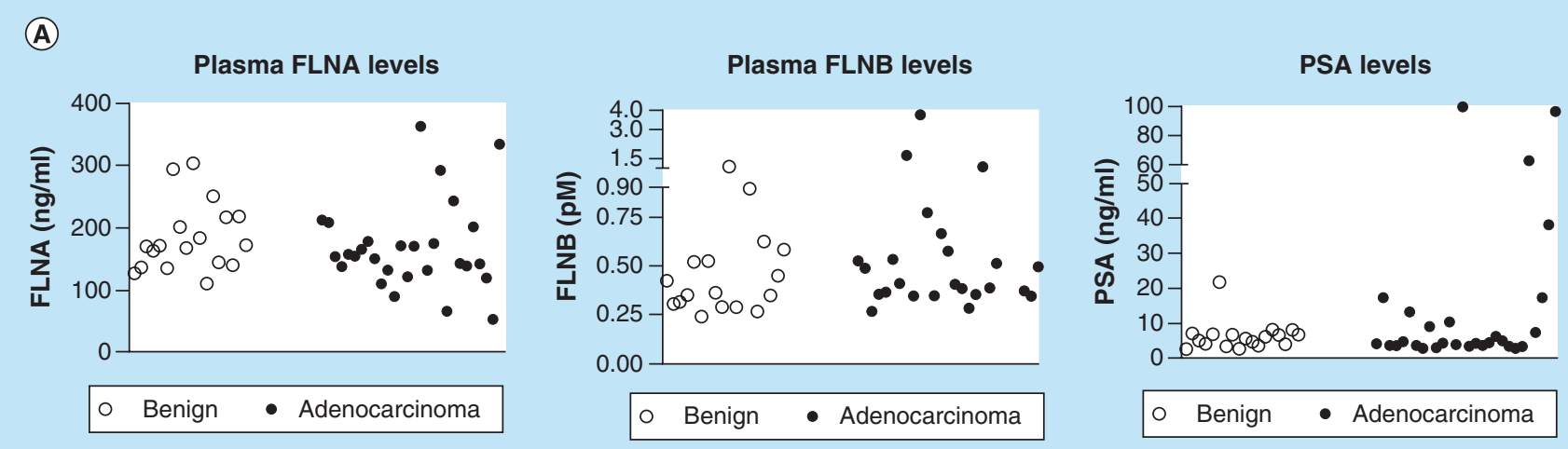

(B)

FLNA vs FLNB in patients with benign neoplasia

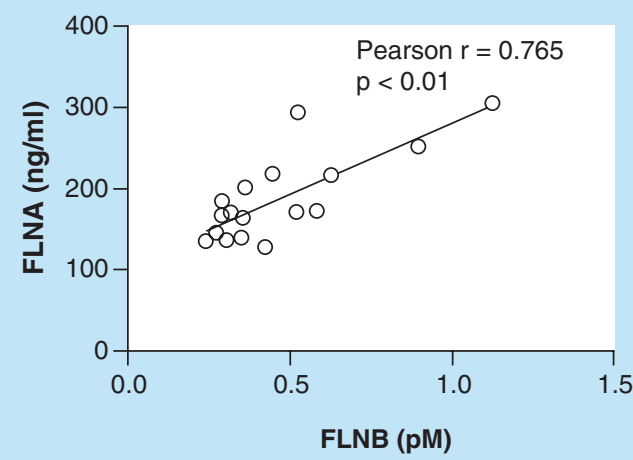

FLNA vs FLNB

in patients with adenocarcinoma

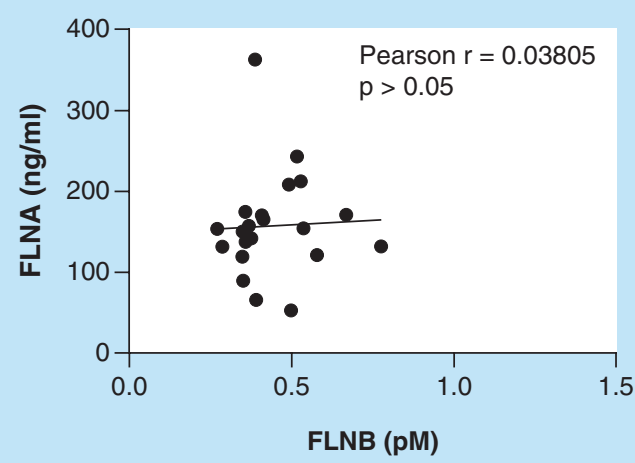

Figure 4. Detection of filamin-A and -B in human plasma. ELISA was used to detect FLNA and FLNB levels in blood samples from patients with suspected prostate cancer. (A) Scatterplot representation of FLNA, FLNB and PSA levels in patients. (B) Correlation analysis of FLNA and FLNB in patients with benign cases or adenocarcinoma.

FLNA: Filamin-A; FLNB: Filamin-B; PSA: Prostate-specific antigen. 
lated in a variety of cancers, including ovarian [30], breast [31] and pancreatic [32]. We note that both FLNA and FLNB were detected in the secretome of pancreatic cell lines, MIA-Paca2 and panc1, and at levels that were not significantly different from $\mathrm{PrCa}$ cells (data not shown). Thus, while FLNA and FLNB alone would not be sufficient to rule out other malignancies, inclusion of FLNA and FLNB in a panel (e.g., similar to the Oncotype DX PrCa assay) in conjunction with standard noninvasive PSA-based tests may potentially be used to diagnose PrCa.

The PSA test is the first-line biomarker option to detect $\mathrm{PrCa}$ [33], and while beneficial it requires a digital rectal exam and follow-up biopsies to confirm diagnosis. PSA is organ-specific and the processing and activation of pro-PSA to PSA requires the activity of human kallikrein proteins (hk2, hk4 and hk15) and serine proteases encoded by chromosome 19 (in humans), factors that contribute toward lack of specificity in diagnosis of prostatic disease [20]. The main advantage of including additional markers in a blood-based panel along with PSA is the reduction in costs that are associated with invasive follow-up biopsies, these include monetary and patient-related quality of care. Thus, development and inclusion of biomarkers unrelated to PSA is critical. In this report the biomarkers identified appear to be independent of PSA levels in vitro and in clinical specimens.

\section{Conclusion}

This report provides evidence to support the use of a novel data-driven strategy to identify causal markers of PrCa. Using a combined in vitro and in silico approach, FLNB and KRT19 were identified as potential biomarkers for PrCa and FLNA was found to be causally linked to FLNB. These data suggest that the combined use of both markers in a panel may be necessary for detecting PrCa.

\section{Future perspective}

The current method for detection of PrCa includes screening with a PSA blood test followed by a digital rectal exam and prostate biopsy to confirm diagnosis. However, due to the lack of specificity of PSA-based tests and the costs associated with overdiagnosis and unnecessary biopsies there is a critical need for novel noninvasive biomarkers for $\mathrm{PrCa}$ that are PSA-independent. The methods described here provide a more rapid approach than traditional methods to identify potential biomarkers of PrCa. In addition, the markers identified here when used in conjunction with other noninvasive blood-based markers in a panel, including PSA, may provide improved predictive power in detecting PrCa. Future efforts should focus on identifying novel biomarkers for PrCa to reduce the burden of overdiagnosis and unnecessary biopsies and associated costs on the patient and healthcare system.

\section{Acknowledgements}

The authors would like to thank E Diamandis, E Klein, S Shrivastava and A Dobi for their critical review of the manuscript and P Tekumalla and E Grund for assisting with preparation.

\section{Author contributions}

NR Narain, AR Diers, A Dalvi, W Wu, VR Akmaev, S Gesta, VK Vishnudas and R Sarangarajan conceived and designed the experiments. AR Diers, S Lao, JY Chan, S Schofield, J Andreazi, R Ouro-Djobo, JJ Jimenez, T Friss, N Tanna, S Wang, D Bunch, Y Sun, K Thapa, S Gesta and LO Rodrigues acquired, analyzed and/or interpreted the data. NR Narain, AR Diers, K Thapa, S Gesta, VK Vishnudas and R Sarangarajan drafted and revised the manuscript critically for important intellectual content. All authors approved the final version to be published.

\section{Financial \& competing interests disclosure}

Funding for all components of this research study was provided by BERG, LLC. JJ Jimenez is a paid advisor for BERG, LLC and has stock options in the company. S Wang and D Bunch are paid advisors for BERG, LLC. S Wang has received grants from Roche Diagnostics. All other authors listed are current or previous employees of BERG, LLC and have stock options. NR Narain is co-founder of BERG, LLC. The authors have no other relevant affiliations or financial involvement with any organization or entity with a financial interest in or financial conflict with the subject matter or materials discussed in the manuscript apart from those disclosed.

No writing assistance was utilized in the production of this manuscript.

\section{Ethical conduct of research}

The authors state that they have obtained appropriate institutional review board approval or have followed the principles outlined in the Declaration of Helsinki for all human or animal experimental investigations. In addition, for investigations involving human subjects, informed consent has been obtained from the participants involved.

\section{Open access}

This work is licensed under the Creative Commons Attribution 4.0 License. To view a copy of this license, visit http://creativecommons.org/licenses/by/4.0/ 
- Proteomic profiling of cancer cells is a promising strategy to identify novel biomarkers for disease.

- Secreted proteins theoretically enter bodily fluids and thus represent an easily available matrix for potential biomarkers that can be detected via noninvasive methods.

- A novel data-driven, systems biology-based approach was used to identify potential biomarkers for prostate cancer.

MethodsFLNA: Filamin-A; FLNB: Filamin-B; KRT19: Keratin-19; PSM: Peptide spectral match;

- The approach used in this study combined in vitro perturbation biology and proteomic analysis with in silico Bayesian causal analysis and delineation of subnetworks.

- The metastatic prostate cancer cell line, PC-3, was exposed to perturbations mimicking the tumor microenvironment (poor oxygenation, low $\mathrm{pH}$ and diminished nutrient state) and conditioned media were subjected to proteomic analysis 24 and $48 \mathrm{~h}$ after culture.

- Proteomic data were analyzed using a Bayesian network inference approach, in which each particular factorization and the choice of parameters are a distinct probabilistic model of the process that created the observed experimental data.

- Subnetworks linked to the functional variables were ranked based on their Burt's constraint score metric, which measures the extent to which nodes (proteins) are connecting to unconnected modules and the relationship redundancy within each of these modules.

- Transcriptional regulation and secretion of the identified biomarkers, Filamin-B (FLNB) and Keratin-19 (KRT19), were characterized in in vitro models to validate the findings. Filamin-A (FLNA), a protein causally linked to FLNB, was also included in the analysis to biologically validate the in vitro and in silico findings.

Results

- FLNB and KRT19 were identified as very important nodes that were causally influenced by culture conditions of hypoxia and lactic acid in the metastatic prostate cell line, PC-3.

- FLNA was found to be causally linked to FLNB.

- The mRNA expression levels of FLNA, FLNB and KRT19 were differentially expressed in a panel of prostate cancer cell lines (LnCAP, DU145 and PC-3) compared to normal human prostate epithelial cells.

- Exposure to prostate cancer-relevant stimuli differentially affects transcriptional regulation of the biomarkers in the panel of cell lines and their regulation appears to be independent of prostate-specific antigen expression.

- Both FLNA and FLNB were found in the secretome of each cancer cell line examined. KRT19 was detected in DU145 and PC-3, but not in LnCAP cell lines.

- Both FLNA and FLNB were detected in plasma from men with suspected prostate cancer.

- In patients with benign cases a strong positive correlation between blood levels of the two markers was observed (Pearson $r=0.765 ; p<0.001$ ).

- In patients with adenocarcinoma the relationship between FLNA and FLNB blood levels was diminished (Pearson $r=0.030805 ; p>0.05$ )

\section{Discussion}

- The use of the secretome provides a surrogate to identify potentially noninvasive markers.

- The secretome includes an array of proteins involved in many processes, including mechanisms important for invasion and metastasis of cancer cells.

- Prior studies have demonstrated a role for FLNA in normal prostate physiology and in prostate cancer.

- This report is the first to identify FLNB as a potential biomarker for prostate cancer.

- The in vitro and in silico findings demonstrate that FLNB is causally associated with FLNA in prostate cancer.

- Clinical data provide preliminary support of a relationship between FLNB and FLNA that is dysregulated in patients with prostate cancer.

\section{Conclusion}

- The findings demonstrate the use of a systems biology approach to identify potential biomarkers for prostate cancer and provide preliminary validation of this approach.

- The combined use of both markers in a panel in conjunction with prostate-specific antigen may potentially yield better predictive power for detection of prostate cancer than prostate-specific antigen alone.

\section{References}

1 Alberts AR, Schoots IG, Roobol MJ. Prostate-specific antigen-based prostate cancer screening: past and future. Int. J. Urol. 22(6), 524-532 (2015).

2 Gardiner RA, Chambers SK, Williams SG, Yaxley J, Samaratunga H, Frydenberg M. Prostate cancer - part one: detection. In: Endotext. De Groot LJ, Beck-Peccoz P, Chrousos G et al. (Eds). South Dartmouth, MA, USA (2000).

3 Moyer VA, Force USPST. Screening for prostate cancer: US Preventive Services Task Force recommendation statement. Ann. Intern. Med. 157(2), 120-134 (2012). 
4 Shtivelman E, Beer TM, Evans CP. Molecular pathways and targets in prostate cancer. Oncotarget 5(17), 7217-7259 (2014).

5 Tjalsma H, Bolhuis A, Jongbloed JD, Bron S, Van Dijl JM. Signal peptide-dependent protein transport in Bacillus subtilis: a genome-based survey of the secretome. Microbiol. Mol. Biol. Rev. 64(3), 515-547 (2000).

6 Ross PL, Huang YN, Marchese JN et al. Multiplexed protein quantitation in Saccharomyces cerevisiae using amine-reactive isobaric tagging reagents. Mol. Cell. Proteomics 3(12), 1154-1169 (2004).

7 Pearl J. Causality, Reasoning and Inference. Cambridge University Press, NY, USA. (2000).

8 Xing H, Mcdonagh PD, Bienkowska J et al. Causal modeling using network ensemble simulations of genetic and gene expression data predicts genes involved in rheumatoid arthritis. PLoS Comput. Biol. 7(3), e1001105 (2011).

9 Burt R, Xa S. Structural holes and good ideas. Am. J. Soc. 110(2), 349-399 (2004).

10 Yang W, Zou L, Huang C, Lei Y. Redox regulation of cancer metastasis: molecular signaling and therapeutic opportunities. Drug Dev. Res. 75(5), 331-341 (2014).

11 Zhang S, Yang C, Yang Z et al. Homeostasis of redox status derived from glucose metabolic pathway could be the key to understanding the Warburg effect. Am. J. Cancer Res. 5(3), 928-944 (2015).

12 Baldassarre M, Razinia Z, Burande CF, Lamsoul I, Lutz PG, Calderwood DA. Filamins regulate cell spreading and initiation of cell migration. PLoS ONE 4(11), e7830 (2009).

13 Bedolla RG, Wang Y, Asuncion A et al. Nuclear versus cytoplasmic localization of filamin A in prostate cancer: immunohistochemical correlation with metastases. Clin. Cancer Res. 15(3), 788-796 (2009).

14 Sun GG, Lu YF, Zhang J, Hu WN. Filamin A regulates MMP-9 expression and suppresses prostate cancer cell migration and invasion. Tumour Biol. 35(4), 3819-3826 (2014).

15 Anastasiadis AG, Stisser BC, Ghafar MA, Burchardt M, Buttyan R. Tumor hypoxia and the progression of prostate cancer. Curr. Urol. Rep. 3(3), 222-228 (2002).

16 De Marzo AM, Platz EA, Sutcliffe $S$ et al. Inflammation in prostate carcinogenesis. Nat. Rev. Cancer 7(4), 256-269 (2007).

17 Lee HY, Yang EG, Park H. Hypoxia enhances the expression of prostate-specific antigen by modifying the quantity and catalytic activity of Jumonji C domain-containing histone demethylases. Carcinogenesis 34(12), 2706-2715 (2013).

18 Mizokami A, Gotoh A, Yamada H, Keller ET, Matsumoto T. Tumor necrosis factor-alpha represses androgen sensitivity in the LNCaP prostate cancer cell line. J. Urol. 164(3 Pt 1), 800-805 (2000).

19 Debelec-Butuner B, Alapinar C, Varisli L et al. Inflammation-mediated abrogation of androgen signaling: an in vitro model of prostate cell inflammation. Mol. Carcinog. 53(2), 85-97 (2014).

20 Lilja H. Biology of prostate-specific antigen. Urology $62(5$ Suppl. 1), 27-33 (2003).

21 Lancashire LJ, Lemetre C, Ball GR. An introduction to artificial neural networks in bioinformatics-application to complex microarray and mass spectrometry datasets in cancer studies. Brief. Bioinform. 10(3), 315-329 (2009).

22 Mendez O, Villanueva J. Challenges and opportunities for cell line secretomes in cancer proteomics. Proteomics Clin. Appl. 9(3-4), 348-357 (2015).

23 Paltridge JL, Belle L, Khew-Goodall Y. The secretome in cancer progression. Biochim. Biophys. Acta 1834(11), 2233-2241 (2013).

24 Jurado J, Fuentes-Almagro CA, Guardiola FA, Cuesta A, Esteban MA, Prieto-Alamo MJ. Proteomic profile of the skin mucus of farmed gilthead seabream (Sparus aurata). J. Proteomics 120, 21-34 (2015).

25 Delbosc S, Haloui M, Louedec L et al. Proteomic analysis permits the identification of new biomarkers of arterial wall remodeling in hypertension. Mol. Med. 14(7-8), 383-394 (2008).

26 Anti-FLNA Antibody (HPA004747). https://atlasantibodiescom/\#!/products/FLNB

27 Guzvic M, Braun B, Ganzer R et al. Combined genome and transcriptome analysis of single disseminated cancer cells from bone marrow of prostate cancer patients reveals unexpected transcriptomes. Cancer Res. 74(24), 7383-7394 (2014).

28 Amaro A, Esposito AI, Gallina A et al. Validation of proposed prostate cancer biomarkers with gene expression data: a long road to travel. Cancer Metastasis Rev. 33(2-3), 657-671 (2014).

29 Loy CJ, Sim KS, Yong EL. Filamin-A fragment localizes to the nucleus to regulate androgen receptor and coactivator functions. Proc. Natl Acad. Sci. USA 100 (8), 4562-4567 (2003).

30 Bandaru S, Zhou AX, Rouhi P et al. Targeting filamin B induces tumor growth and metastasis via enhanced activity of matrix metalloproteinase-9 and secretion of VEGF-A. Oncogenesis 3, e119 (2014).

31 Tian HM, Liu XH, Han W, Zhao LL, Yuan B, Yuan CJ. Differential expression of filamin A and its clinical significance in breast cancer. Oncol. Lett. 6(3), 681-686 (2013).

32 Li C, Yu S, Nakamura F et al. Binding of pro-prion to filamin A disrupts cytoskeleton and correlates with poor prognosis in pancreatic cancer. J. Clin. Invest. 119(9), 2725-2736 (2009).

33 Sartori DA, Chan DW. Biomarkers in prostate cancer: what's new? Curr. Opin. Oncol. 26(3), 259-264 (2014). 